\title{
OP12
}

\section{THE INFLUENCE OF ROAD LIGHTING ON CYCLIST NUMBERS AND SAFETY}

\author{
Steve Fotios et al.
}

DOI 10.25039/x46.2019.OP12

from

CIE x046:2019

\author{
Proceedings \\ of the \\ 29th CIE SESSION \\ Washington D.C., USA, June 14 - 22, 2019 \\ (DOI 10.25039/x46.2019)
}

The paper has been presented at the 29th CIE Session, Washington D.C., USA, June 14-22, 2019. It has not been peer-reviewed by CIE.

\section{(C) CIE 2019}

All rights reserved. Unless otherwise specified, no part of this publication may be reproduced or utilized in any form or by any means, electronic or mechanical, including photocopying and microfilm, without permission in writing from CIE Central Bureau at the address below. Any mention of organizations or products does not imply endorsement by the CIE.

This paper is made available open access for individual use. However, in all other cases all rights are reserved unless explicit permission is sought from and given by the CIE.

CIE Central Bureau

Babenbergerstrasse 9

A-1010 Vienna

Austria

Tel.: +43 17143187

e-mail: ciecb@cie.co.at

www.cie.co.at 


\title{
THE INFLUENCE OF ROAD LIGHTING ON CYCLIST NUMBERS AND SAFETY
}

\author{
Fotios, S. ${ }^{1}$, Uttley, J. ${ }^{1}$, Bohm, A. ${ }^{2}$, Qasem, H. ${ }^{1}$ \\ ${ }^{1}$ University of Sheffield, Sheffield, UNITED KINGDOM, ${ }^{2}$ University of Lincoln, Lincoln, UNITED \\ KINGDOM \\ j.uttley@sheffield.ac.uk
}

DOI $10.25039 / \times 46.2019 .0 P 12$

\begin{abstract}
Lighting can play an important role in encouraging cycling after-dark and making it safer. This paper describes ongoing research to establish a basis for design guidance when lighting for cyclists. Comparison of cyclist counts and estimated illuminance levels suggest a small increase in illuminance after-dark can significantly reduce the negative impact darkness has on cycling rates. Experimental work investigating obstacle detection by cyclists reveals that cycle lighting may not provide any benefit for detecting obstacles on lit roads and may even make detection worse, with the vertical position of the front cycle lamp being important. Cycle lamps also serve the purpose of making cyclists more visible but drivers often fail to detect cyclists even when they are highly visible. Lighting should therefore be considered alongside other approaches to cyclist safety, one of which is introducing presumed liability as a legal consideration to increase driver's attention for cyclists.
\end{abstract}

Keywords: Cycling, Road safety, Cycle lighting, Road lighting

\section{Background}

Cycling provides a range of health and environmental benefits that have led many Governments to promote it as a mode of transport (Public Health England, 2016). Despite these efforts cycling rates remain low in many countries. In the UK for example, less than $2 \%$ of trips are cycled, despite $68 \%$ of trips being less than 5 miles (Department for Transport, 2018a). A major deterrent to people cycling is its perceived safety, with many people being put off by risks they associate with cycling on the road such as being involved in a collision with a motor vehicle (Winters et al., 2011). Such perceived risks are supported by reality, with cyclists experiencing greater risk of involvement in a collision than drivers, and are more likely to be killed if they are involved in a collision (Department for Transport, 2018b).

Darkness may exaggerate the perceived and real risks of cycling, potentially leading to fewer people cycling after-dark and an increase in road traffic collisions (RTCs). Only $23 \%$ of women and $36 \%$ of men in the UK felt safe cycling during hours of darkness (Sustrans, 2018a). This may be due to feeling at greater risk of being victimised when it is dark; we know that pedestrians feel a lower degree of reassurance during hours of darkness (Fotios et al., 2018) and there is also evidence that female cyclists also feel less safe from crime and antisocial behaviour at night - data from a survey in Glasgow suggested only $36 \%$ of women felt safe cycling at night compared with $75 \%$ during the day (Sustrans, 2018b). Reduced visibility afterdark may also increase the perceived risk of getting injured because it is both harder to see and harder to be seen. Lower light levels make it more difficult to see and (and hence avoid) a hazard in the path at night (Uttley et al., 2017; Bullough \& Rea, 2000). Drivers also find it more difficult to detect a cyclist at night (Wood et al., 2010). Reduced visibility contributes significantly to the increased collision risk for cyclists at night (Twisk \& Reurings, 2013). Records of travel flow rates confirm that fewer people cycle when it is dark compared with daylight, even after accounting for the time of day and year (Uttley \& Fotios, 2017; Fotios et al., 2019)

Lighting has potential to make cyclists feel safer, encourage them to cycle after-dark and reduce the risk of their involvement in an RTC, through increasing reassurance and visibility. Current lighting standards (e.g. British Standards Institution, 2012) tend to group the lighting requirements of cyclists and pedestrians together, for example the P-class lighting specifications are designed for both pedestrians and cyclists, with no distinction. Assuming these two types of traveller have the same lighting requirements may be incorrect however. In 
the UK for example, cyclists are legally required to use lights fixed to the front and rear of their bike. Cyclists also travel at faster speeds than pedestrians, warranting different infrastructure design considerations (Parkin \& Rotherham, 2010). Importantly for lighting, this means they need to detect hazards at a greater distance than pedestrians, and the type of hazard requiring detection may also vary, based on the potential consequences for a pedestrian versus a cyclist. The amount of separated cycling infrastructure is also low in most countries and cyclists often share the road with vehicles, meaning a greater need to be visible to drivers and less opportunity to avoid road hazards. Existing lighting regulations and recommendations do not adequately reflect the needs of cyclists and there is considerable potential for ensuring lighting is fit for the purposes of cycling (Fotios \& Castleton, 2017).

The lighting standards that support pedestrian road lighting have little empirical basis (Fotios \& Gibbons, 2018) and work is ongoing in CIE TC 4-52 to establish the evidence needed to inform appropriate standards. Similar work is required in parallel to provide evidence about the lighting required to support cycling and make it a safe mode of transport. This paper describes ongoing research aimed at addressing this goal. We consider the influence of lighting on the confidence to cycle and the ability of the cyclist to detect hazards. We also discuss responsibilities of road users to promote safety, as it is important to be aware of what lighting can and cannot influence when it comes to cyclist safety.

\section{Lighting and the confidence to cycle}

Previous research has demonstrated a link between ambient light levels and cycling rates (Uttley \& Fotios, 2017; Fotios et al., 2019). These studies used observational count data from the United States to assess the impact of darkness on the number of people cycling. A number of factors are likely to co-vary with darkness and also influence the decision to cycle, two of the most significant being time of day and time of year (Aultman-Hall et al., 2009). For example, there are more hours of darkness during winter months than summer months, and the weather conditions during these two periods of the year will strongly influence the number of people choosing to cycle. To overcome these potentially confounding factors the two studies by Uttley and colleagues used an odds ratio method of analysis. This compares counts of cyclists in the same hour of the day but when it is in either daylight or darkness. This ratio is then contrasted against counts over the same two periods of the year but during a 'control' hour where the ambient light remained constant (always daylight, or always darkness, regardless of the time of year). The resulting odds ratio quantifies the effect of darkness on cycling rates, with a larger odds ratio indicating a greater reduction in cyclists associated with darkness. Fotios et al. (2019) found an odds ratio for cyclists of 1.67, suggesting people were 1.67 times more likely to cycle during daylight than after-dark, after accounting for time of day and seasonal factors.

Lighting may reduce the negative impact of darkness on the number of people cycling. To assess this, a large sample of cyclist counts was obtained from 48 automated counters located across the city of Birmingham, UK, for the period 2012 to 2015. An odds ratio was calculated for each of these 48 locations, providing a measure of how large the reduction in cyclists afterdark was in those locations. See Equation 1.

$$
\text { Odds ratio }_{i}=\frac{\text { Case }_{\text {day }, i} / \text { Case }_{\text {dark }, i}}{\text { Control day }, i / \text { Control }_{\text {dark }, i}}
$$

where for counter location $i$
Case dark
is the count of cyclists in the case hour when it is dark
Case $_{\text {day }}$
is the count of cyclists in the case hour when it is in daylight
Controldark $^{2}$
is the count of cyclists in control hours when the case hour is dark
Controlday
is the count of cyclists in control hours when the case hour is in daylight 
These 48 odds ratios were compared against an indicative measure of average illuminance at the associated counter location. This measure of illuminance was estimated from night-time aerial imagery recorded by the UK Environment Agency in 2009. Night-time aerial images of Birmingham were resampled and converted to a greyscale image, following the procedure set out by Hale et al. (2013). Previous work had confirmed pixel intensity values in these images were strongly predictive of actual illuminances measured on the ground $\left(R^{2}=0.91\right.$ ) (Hale et al., 2013). A $15 \mathrm{~m}$ buffer was drawn around the road or path section that each counter was located on using QGIS software (QGIS Development Team, 2018), and the average pixel value within this buffer was calculated. Using the previous mapping of pixel values to illuminances carried out by Hale et al., the average pixel value was converted to an estimated average illuminance for each location. These are plotted against the odds ratios calculated for the same locations in Figure 1.

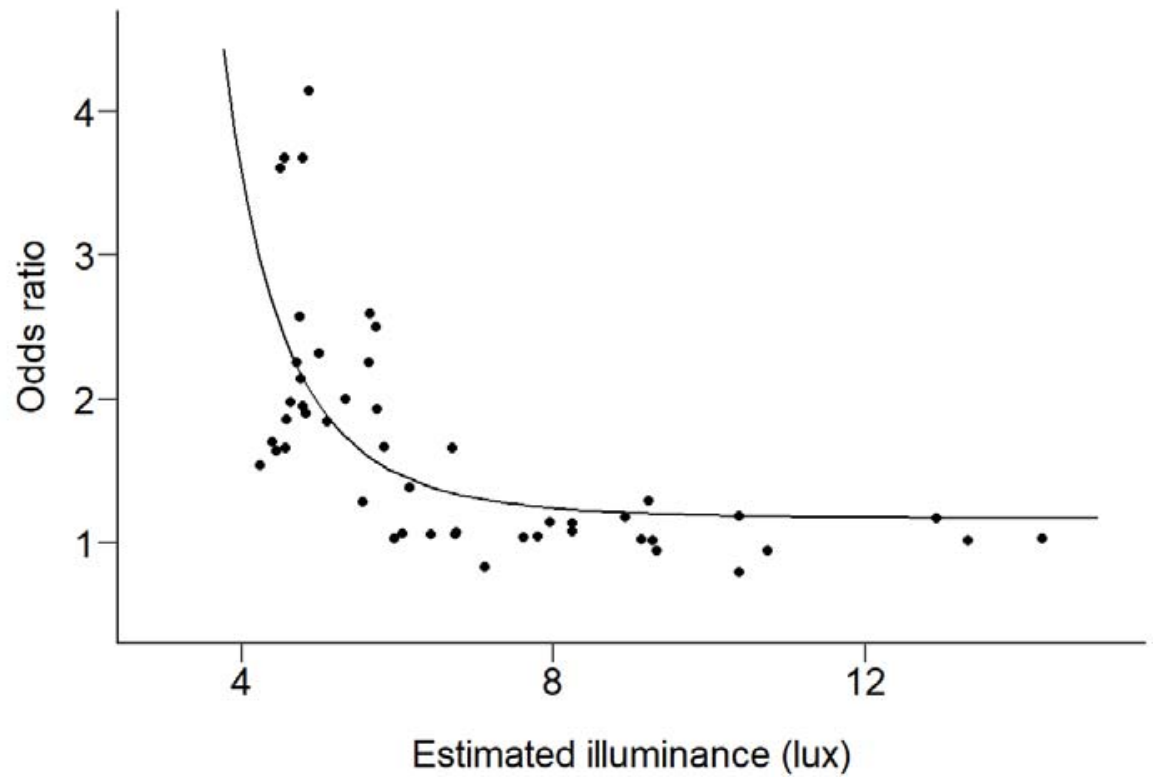

Figure 1 - Odds ratio quantifying effect of darkness on cycling rates compared against estimated illuminance at 48 counter locations in Birmingham, UK. Larger odds ratio indicates bigger reduction in cyclists due to darkness. Illuminance estimated from pixel intensities in night-time aerial imagery. Regression line calculated using formula: Odds ratio $=1.17+2450\left(1 /\right.$ Illuminance $\left.{ }^{5}\right)$.

There is a clear non-linear relationship between the illuminance at a location and the change in cycling rates after-dark. At low light levels, up to around 6 lux, a small increase in illuminance produces a large drop in the odds ratio. This effect reaches a plateau at an average illuminance of 6 lux, with further increases in illuminance above this making little difference to the odds ratio. The implication is that only small increases in illuminance above a minimum baseline level are needed to encourage more people to cycle after-dark. Further additional increases may be unwarranted, if the goal is to further encourage people to cycle during hours of darkness.

Encouraging cycling after-dark is not the only reason for providing lighting however. Another reason is to enable cyclists to detect hazards in their path. The next section presents experimental work to assess the impact of lighting on a cyclist's performance at detecting obstacles in the path.

\section{Obstacle detection by cyclists}

The illuminance and spectrum of road lighting influences a pedestrian's ability to detect an obstacle in their path (e.g. Uttley et al., 2017). The influence lighting has on detection of obstacles by cyclists is likely to be different to pedestrians however. Cyclists travel at faster speeds than pedestrians and have different cognitive demands due to the stability and safety requirements of cycling. Cyclists in the UK and many other countries are also required to use both front and rear cycle lamps after-dark. Although one of the purposes of these cycle lamps 
is to make the cyclist more visible to other road users, another purpose for the front light is to illuminate the path ahead to aid hazard detection. It is possible however that the light from the front cycle lamp and any road lighting nearby could work against each other in terms of reducing the contrast provided by an obstacle that is essential to its detection. This is due to the directionality of the two light sources, road lighting being predominantly vertical to reveal hazards by negative contrast against the background and cycle lighting being predominantly horizontal to reveal hazards by positive contrast. The contribution of light from a cycle lamp to obstacle detection performance, and its interaction with light from road lighting, may also be influenced by the position of the cycle lamp. Conventionally front cycle lamps are fixed to the handlebars. Alternative vertical positions are possible though, for example at the hub of the front wheel or on the cyclist's head or helmet. It is not currently known how the vertical position of the cycle lamp influences obstacle detection, or how the light provided interacts with that provided by road lighting. Three laboratory experiments were carried out to investigate how variations in the intensity of cycle and road lighting, and the position of the cycle lamp, influence obstacle detection. Selected results from these experiments are presented. Extended details about methods and results from all experiments carried out as part of this study can be found in Fotios et al. (2017).

Participants sat on a static bicycle at the open end of a three-sided chamber with walls covered in black cloth (see Figure 2). A $200 \mathrm{~mm}$ cylinder was positioned at the centre of the chamber, within a false floor painted in Munsell N5 grey paint. This cylinder could be raised above the surface of the false floor using electric motors to simulate an obstacle in the path of a cyclist. This obstacle was approximately $2.6 \mathrm{~m}$ from the participant's position, giving an eye-to-obstacle distance of approximately $3 \mathrm{~m}$. The obstacle could be raised to a maximum of $28.4 \mathrm{~mm}$, at speeds of either 1 or $2 \mathrm{~mm} / \mathrm{s}$.

It was important to limit as much as possible participants looking directly at the area where the obstacle was, as hazard detection is primarily a task for peripheral vision. A dynamic fixation target (a ' + ' symbol) was therefore projected on to the far wall of the chamber. This target randomly moved within a $1.05 \times 2.00 \mathrm{~m}$ ellipse. It would also change to a single digit between 1 and 9 at random intervals between 2 and $6 \mathrm{~s}$, for $0.2 \mathrm{~s}$, before changing back to the target symbol. Participants were asked to track the dynamic target and state aloud the digit they saw each time it changed. This task was designed to hold the participant's foveal attention, ensuring the floor obstacle was in peripheral vision (Fotios et al., 2016).

The chamber was lit from above by two LED arrays. These provided light with an S/P ratio of 1.6. Three illuminance conditions were tested: $0.2,2.0$ and 20.0 lux (as measured at centre of top surface of obstacle). A fourth condition of no overhead lighting (LED arrays switched off) was also included. A second LED light source simulated a forward-facing cycle lamp. This was positioned at one of three possible heights above the floor $-565 \mathrm{~mm}$ (hub-mounted); $1370 \mathrm{~mm}$ (handlebar-mounted); and $1830 \mathrm{~mm}$ (helmet-mounted). The light from the cycle lamp had an $\mathrm{S} / \mathrm{P}$ ratio of 2.1 , with luminances of $0.1,0.32$ and $1.0 \mathrm{~cd} / \mathrm{m}^{2}$ used, as measured on the side of the raised obstacle facing the participant. A fourth condition of cycle lamp switched off was also included.

Ten participants took part in each of the three experiments (18 females, mean age $=26$ years). Participants were asked to pedal the static bicycle at a constant rate, with an alarm sounding if cadence dropped below 50 rotations per minute or above 80 rotations per minute. They were told their primary task was to follow the dynamic fixation target on the far wall and read aloud any digits they saw, but to also a press a button located on the handlebar as soon as they noticed the obstacle appear in their peripheral vision. Three experiments were carried out, exploring different combinations of lighting conditions. Experiment 1 used only one cycle lamp position (handlebar-mounted) but compared the four cycle lamp luminance conditions and four overhead illuminance conditions. Experiment 2 used all three cycle lamp positions, all four overhead illuminance conditions, but only one cycle lamp luminance $\left(0.32 \mathrm{~cd} / \mathrm{m}^{2}\right)$. Experiment 3 repeated experiment 1 , but with the cycle lamp in the hub-mounted position rather than handlebar-mounted. 

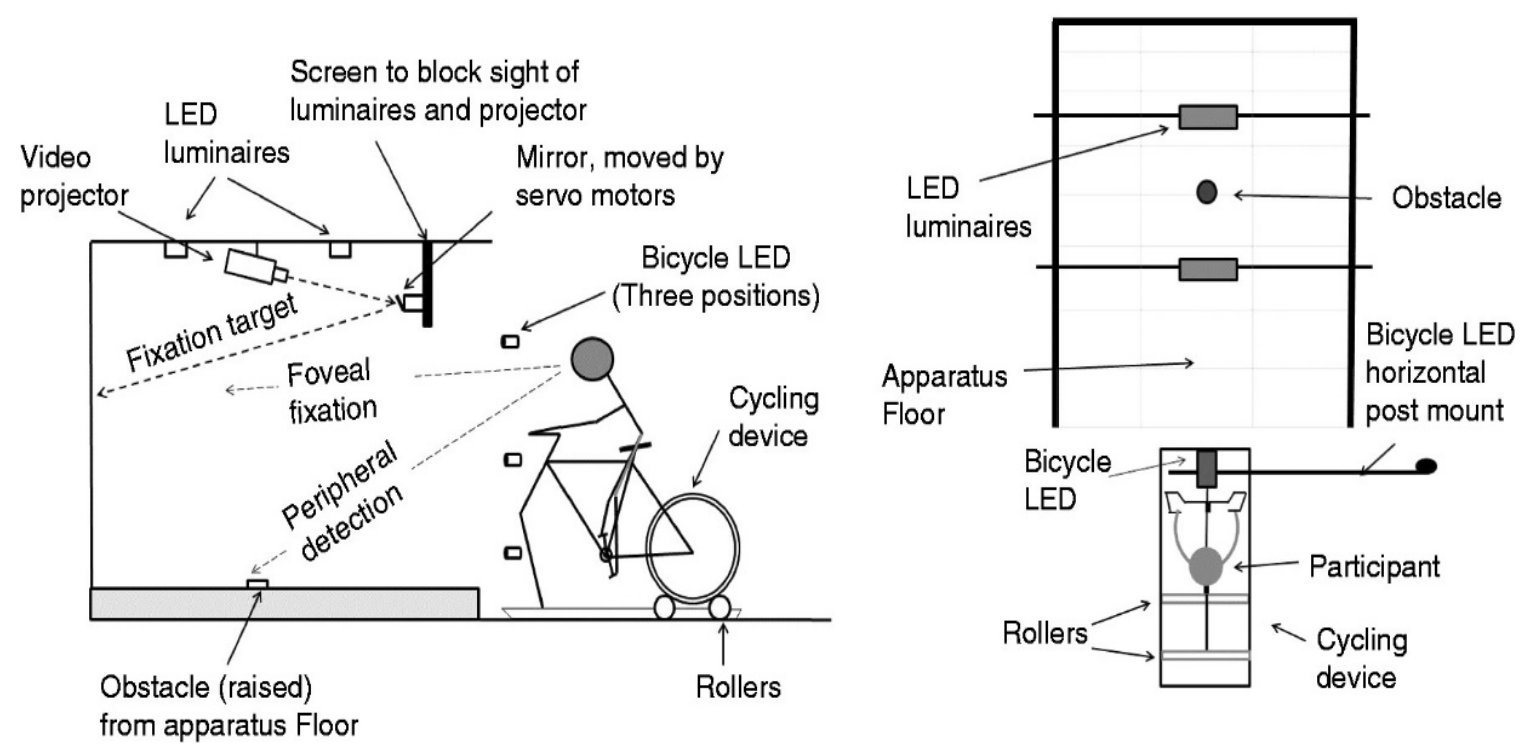

Figure 2 - Cyclist obstacle detection experiment apparatus. Taken from Fotios et al. (2017).

Figure 3 shows results from all three experiments. There was no benefit of the handlebarmounted cycle lamp being switched on at all three overhead illuminances (Figure 3a). Switching on the cycle lamp actually led to worse detection performance at the lower two overhead illuminances. When the cycle lamp was switched off, the increase in illuminance from 0.2 to 2.0 lux significantly improved detection performance, but this effect plateaued from 2.0 to 20.0 lux. Comparing the three cycle lamp positions (Figure $3 \mathrm{~b}$ ), the hub-mounted lamp led to best detection performance. Statistical tests (Wilcoxon signed-rank) confirmed the median detection height was significantly lower for the hub-mounted condition compared with the other two positions, but only at the lowest illuminance level. At 2.0 and 20.0 lux there were no significant differences between the three cycle lamp positions. Experiment 3 (Figure 3c) confirmed the potential benefits of hub-mounted cycle lighting. Using the hub lamp at the lowest overhead illuminance level significantly improved detection performance, in contrast to using the handlebar lamp. However, at the highest overhead illuminance, use of the hub lamp significantly worsened detection performance.

The obstacle appeared twice in each condition, rising at two different speeds (1 and $2 \mathrm{~mm} / \mathrm{s}$ ). When a participant pressed the response button to indicate detection of the obstacle, its height at that point was recorded. The mean height across the two trials in each condition was used as the dependent variable.

Two key points can be taken from the results of these experiments. First, use of a cycle lamp on a lit road frequently offers no additional benefits for obstacle detection and may even make it harder to see an obstacle. Second, the position of the cycle lamp matters. At low overhead illuminances ( $\leq 0.2$ lux) a hub-mounted lamp improved detection over a handlebar-mounted lamp. This improvement was enough to offset the drop in detection performance that would normally be seen at these low overhead illuminances, compared with higher illuminances, if using a conventional handlebar-mounted lamp. These two key points are both linked to the contrast between the obstacle and its surrounding surface, and how this changes depending on the interaction between overhead and cycle lamp lighting, and the position of the cycle lamp. 


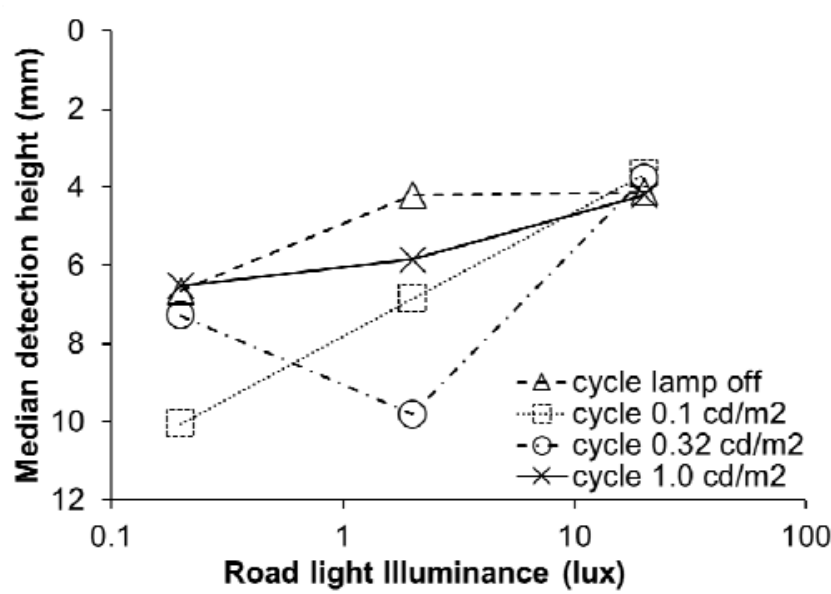

(a)

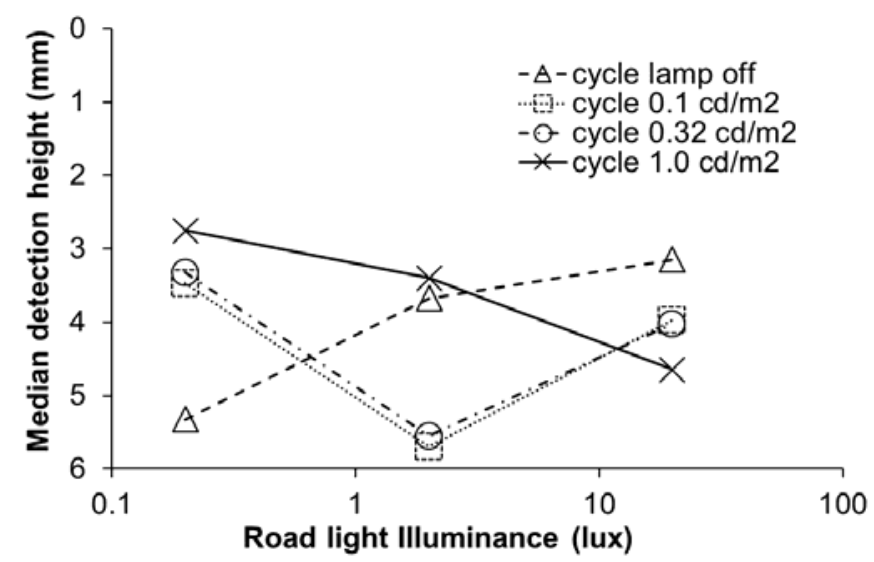

(c)

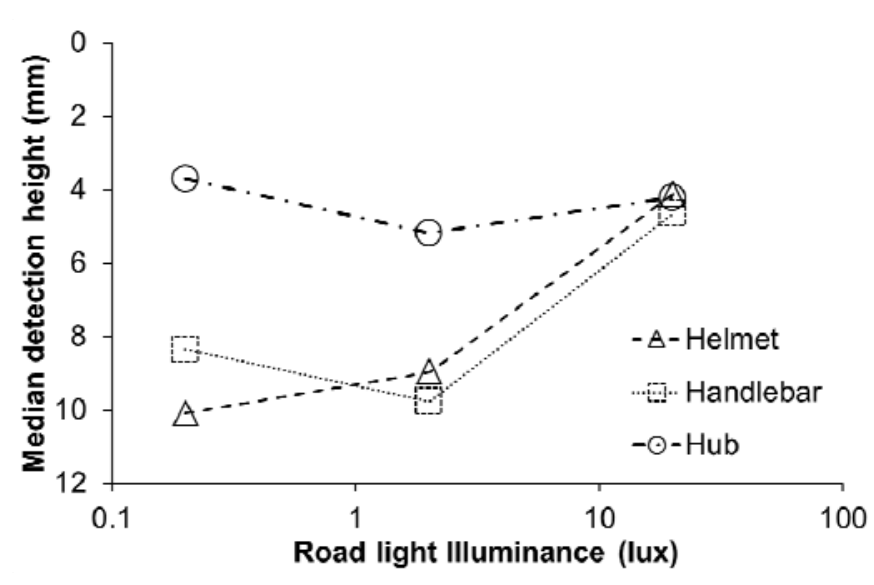

(b)

Figure 3 - Median detection heights at different overhead illuminances for (a) Experiment 1, comparing different luminances from handlebar-mounted cycle lamp; (b) Experiment 2, comparing different cycle lamp positions (luminance $=0.32 \mathrm{~cd} / \mathrm{m}^{2}$ ); (c) Experiment 3, comparing different luminances from hub-mounted cycle lamp. Note that $y$-axis scale has been reversed as smaller median detection height indicates better performance.

\section{Road user's responsibilities}

The three experiments reported in section 3 focused on the role of cycle lamps in helping the cyclist to see hazards. Cycle lamps are also important in helping the cyclist be seen by other road users. Although the use of cycle lamps is often recommended or mandatory after-dark, surveys show they are not always used (McGuire \& Smith, 2000). Even assuming they are used, being visible is not the only determinant of whether a cyclist will be detected (and avoided) by another road user. This is demonstrated by 'looked but failed to see' incidents, where a driver can look directly at a cyclist without realising they are there (Herslund \& Jorgensen, 2003). Such errors imply that even if a cyclist is visible they may not be detected because the driver's attention is influenced by top-down, non-visual processes such as what they expect to see and the value they put on detecting different things. So whilst cyclists must be responsible for using cycle lamps there should also be recognition that cycle lamps are not the only solution to ensuring cyclists are seen by drivers after-dark. Promoting a responsibility amongst drivers to look out for cyclists may have an effect on reducing collisions.

Collisions between cyclists and drivers tend to result in greater injury for the cyclist than the driver. Cyclists are aware of this and tend to look out for motorised vehicles. Drivers are less aware of cyclists, partly because they pose less risk than other motorised vehicles, and also because they are encountered less frequently and therefore may not be prominent in the driver's expectations about what they are likely to see (Tin Tin et al., 2014). A similar reduced 
awareness occurs for motorcyclists. Research using photographs of driving situations showed that an additional stimulus was twice as likely to be missed by an observer if it was a motorcycle compared with a taxi (Pammer et al., 2018). Experience of cycling can increase a driver's awareness for other cyclists on the road, leading to a greater chance a cyclist will be detected (Beanland \& Hansen, 2017). Roge et al. (2017) demonstrated that motorists who also cycle had fewer collisions with cyclists in a driving simulator task than non-cycling motorists, and also detected cyclists at a greater distance. Increasing a driver's awareness of and attention for cyclists can therefore reduce collisions between drivers and cyclists, but this cannot be achieved by road or cycle lighting alone. An alternative approach could involve changing the personal consequences to drivers of being involved in a collision with a cyclist.

In many common law jurisdictions, including the UK and the United States, the law regulating driver-cyclist collisions is fault-based. This means a cyclist victim of a collision needs to prove that the driver has been careless and that this carelessness caused the victim's injuries. The amount of compensation awarded to victims is reduced if their own carelessness contributed to their injuries (Law Reform (Contributory Negligence) Act 1945). Most cases of this sort that reach court (rather than being settled by insurance companies before litigation commences) involve very clear poor behaviour by drivers, such as ignoring give-way or stop signs at junctions, so it is not certain whether a 'looked but failed to see' collision would be considered to be the result of driver carelessness, or whether reasonably competent, non-careless drivers would fail to see cyclists at certain points. Analysis of criminal prosecutions for dangerous or careless driving suggests that courts look generously upon very poor standards of driver behaviour (e.g. Bez, 2017). A cyclist's failure to make use of the legally required form of lighting would very likely result in a reduction of compensation based on the cyclist's own carelessness.

In those jurisdictions operating this 'fault-based' system of prosecution, a move towards 'presumed liability' (also known as 'strict liability') could improve cyclist safety by increasing a driver's attentional awareness of cyclists. Presumed liability would shift the cost of involvement in a collision to the driver. They would need to prove that the cyclist was at fault or would otherwise have to bear all costs associated with the collision. Because many countries with presumed liability regimes also tend to have better cycling infrastructure, it is difficult to be certain how much the legal regime contributes to cyclist safety. But placing the burden of proof on the driver increases the cost of colliding with a cyclist and therefore may make drivers look more for cyclists. This would increase the safety of cyclists and potentially also encourage more people to cycle (Maker, 2015).

\section{Conclusion}

The range of benefits of cycling mean it should be widely encouraged and made as safe as possible. The various strands of research reported in this paper attempt to address these goals in the context of lighting. Section 2 highlighted how light levels strongly influence the decision to cycle or not, and fewer people cycle when it is dark compared with daylight. A minimal increase in light levels can significantly reduce the negative impact of darkness on cycling rates, although the optimal lighting conditions for encouraging cycling have yet to be established. Lighting design standards that enable cyclists to adequately detect hazards after-dark have also yet to be empirically established although the work reported in Section 3 provides some initial results towards that end. These results also show the design and use of cycle lamps for detecting hazards needs careful consideration, including how their light interacts with the vertical light of overhead road lighting, and the position of the lamp on the bicycle or its rider. Finally the findings are a reminder that lighting is not the only answer to improving road safety: lighting must be considered alongside legal consideration which may in turn promote the responsibility of drivers to look for cyclists. 


\section{References}

AULTMAN-HALL, L., LANE, D., \& LAMBERT, R, 2009. Assessing impact of weather and season on pedestrian traffic volumes. Transportation Research Record: Journal of the Transportation Research Board, (2140), 35-43.

BEANLAND, V. AND HANSEN, L.J., 2017. Do cyclists make better drivers? Associations between cycling experience and change detection in road scenes. Accident Anal. Prev., $106,420-427$.

BEZ, 2017. The Incompetence Paradox. Available online: https://beyondthekerb.org.uk/theincompetence-paradox/ [Accessed: 23/04/2019].

BRITISH STANDARDS INSTITUTION, 2012. BS 5489-1: 2013 Code of Practice for the Design of Road Lighting. London: BSI.

BULLOUGH, J.D., REA, M.S. 2000. Simulated driving performance and peripheral detection at mesopic and low photopic light levels. Lighting Res. Technol., 32(4), 194-198.

DEPARTMENT FOR TRANSPORT, 2018a. Table NTS0308. Average number of trips by trip length and main mode: England, 2017. Available online: https://assets.publishing.service.gov.uk/government/uploads/system/uploads/attachment_ data/file/729502/nts0105.ods [Accessed 11/04/2019].

DEPARTMENT FOR TRANSPORT, 2018b. Reported road casualties in Great Britain: 2017 annual report. London: Department for Transport.

FOTIOS, S., UTTLEY, J. AND CHEAL, C., 2016. Maintaining foveal fixation during a peripheral detection task. Lighting Res. Technol., 48(7), 898-909.

FOTIOS, S. AND CASTLETON, H.F., 2017. Lighting for cycling in the UK-A review. Lighting Res. Technol., 49(3), 381-395.

FOTIOS, S., QASEM, H., CHEAL, C. AND UTTLEY, J., 2017. A pilot study of road lighting, cycle lighting and obstacle detection. Lighting Res. Technol., 49(5), 586-602.

FOTIOS, S. AND GIBBONS, R., 2018. Road lighting research for drivers and pedestrians: The basis of luminance and illuminance recommendations. Lighting Res. Technol., 50(1), 154186.

FOTIOS, S., MONTEIRO, A.L. AND UTTLEY, J., 2018. Evaluation of pedestrian reassurance gained by higher illuminances in residential streets using the day-dark approach. Lighting Res. Technol.. Advance online publication, doi: 1477153518775464.

FOTIOS, S., UTTLEY, J. AND FOX, S., 2019. A whole-year approach showing that ambient light level influences walking and cycling. Lighting Res. Technol., 51(1), 55-64.

HALE, J. D., DAVIES, G., FAIRBRASS, A. J., MATTHEWS, T. J., ROGERS, C. D., \& SADLER, J. P., 2013. Mapping lightscapes: spatial patterning of artificial lighting in an urban landscape. Plos One, 8(5), e61460.

HERSLUND, M. B., \& JØRGENSEN, N. O., 2003. Looked-but-failed-to-see-errors in traffic. Accident Anal. Prev., 35(6), 885-891.

LAW REFORM (CONTRIBUTORY NEGLIGENCE) ACT 1945. Available online: http://www.legislation.gov.uk/ukpga/1945/28/pdfs/ukpga_19450028_en.pdf [Accessed 25/04/2019].

MAKER, C., 2015. Strict liability in cycling laws to ready the roads for environmentally friendly commuting. Boston College Environmental Affairs Law Review, 42(2), 473-505.

MCGUIRE, L. AND SMITH, N., 2000. Cycling safety: injury prevention in Oxford cyclists. Inj. Prev., 6(4), 285-287.

PARKIN, J. AND ROTHERAM, J., 2010. Design speeds and acceleration characteristics of bicycle traffic for use in planning, design and appraisal. Transp. Policy, 17(5), 335-341.

PUBLIC HEALTH ENGLAND. 2016. Health matters: Getting every adult active every day. London: Public Health England. 
QGIS DEVELOPMENT TEAM, 2018. QGIS Geographic Information System, version 2.18. Open Source Geospatial Foundation Project. http://qgis.osgeo.org.

ROGÉ, J., NDIAYE, D., AILLERIE, I., AILLERIE, S., NAVARRO, J. AND VIENNE, F., 2017. Mechanisms underlying cognitive conspicuity in the detection of cyclists by car drivers. Accident Anal. Prev., 104, 88-95.

SUSTRANS, 2018a. BIKElife All cities publication. Women: reducing the gender gap. Available online:

https://www.sustrans.org.uk/sites/default/files/file_content_type/bikelife18_reducinggende rgap_digital_2018_07_02.pdf [Accessed 11/04/2019].

SUSTRANS, 2018b. "Are we nearly there yet?" Exploring gender and active travel. Bristol: Sustrans.

PAMMER, K., SABADAS, S. AND LENTERN, S., 2018. Allocating attention to detect motorcycles: The role of inattentional blindness. Hum. Factors, 60(1), 5-19.

TIN TIN, S., WOODWARD, A. AND AMERATUNGA, S., 2014. The role of conspicuity in preventing bicycle crashes involving a motor vehicle. Eur. J. Public Health, 25(3), 517-522.

TWISK, D.A.M. AND REURINGS, M., 2013. An epidemiological study of the risk of cycling in the dark: The role of visual perception, conspicuity and alcohol use. Accident Anal. Prev., $60,134-140$.

UTTLEY, J. AND FOTIOS, S., 2017. Using the daylight savings clock change to show ambient light conditions significantly influence active travel. J. Environ. Psychol., 53, 1-10.

UTTLEY, J., FOTIOS, S. AND CHEAL, C., 2017. Effect of illuminance and spectrum on peripheral obstacle detection by pedestrians. Lighting Res. Technol., 49(2), 211-227.

WINTERS, M., DAVIDSON, G., KAO, D. AND TESCHKE, K., 2011. Motivators and deterrents of bicycling: comparing influences on decisions to ride. Transportation, 38(1), 153-168.

WOOD, J.M., TYRRELL, R.A., MARSZALEK, R.P., LACHEREZ, P.F., CARBERRY, T.P., CHU, B.S. AND KING, M.J., 2010. Cyclist visibility at night: perceptions of visibility do not necessarily match reality. Journal of the Australasian College of Road Safety, 21(3), 5660. 